Supporting Information

\author{
A Highly Efficient Enantioselective Approach to the Securinega Alkaloids: Total \\ Synthesis of (-)-Norsecurinine \\ Ramon Alibès, Pau Bayón, Pedro de March, Marta Figueredo*, Josep Font, Elena García-García \\ and David González-Gálvez \\ Departament de Química, Universitat Autònoma de Barcelona 08193 Bellaterra, Spain \\ marta.figueredo@uab.es
}

Index

General Procedures S2

Preparation of compound 11

S3

Preparation of compound 12

S3

Preparation of compound 13

S5

Preparation of compound 14a

S6

Preparation of compound 15a

S7

Preparation of compound 16

S8

Preparation of compound 17

S9

Preparation of compound 18

S10

(-)-Norsecurinine

S11

${ }^{1}$ HNMR spectra of compounds 12, 14a, 15a, 16, 17 and (-)-3

S13

${ }^{1}$ HNMR spectra of diastereomeric Mosher esters of compound 11 
General Procedures. All reactions were carried avoiding moisture by standard procedures and under nitrogen or argon atmosphere. Commercially available reagents were used as received. The solvents were dried by distillation over the appropriate drying agents. Reactions were monitored by analytical thin-layer chromatography (TLC) using silica gel $60 \mathrm{~F}_{254}$ pre-coated aluminum plates (0.25 mm thickness). Flash column chromatography was performed using silica gel $60 \AA$, particle size 35-70 $\mu \mathrm{m} .{ }^{1} \mathrm{HNMR}$ spectra were recorded on Bruker AC250 (250 MHz) and Bruker ARX400 $(400 \mathrm{MHz})$ spectrometers. Proton chemical shifts are reported in ppm $(\delta)\left(\mathrm{CDCl}_{3} \delta 7.26 \mathrm{ppm}\right)$. ${ }^{13} \mathrm{CNMR}$ spectra were recorded on a Bruker AC250 (62.5 MHz) spectrometer with complete proton decoupling. Carbon chemical shifts are reported in ppm $(\delta)\left(\mathrm{CDCl}_{3}, \delta 77.0\right)$. NMR signals were assigned with the help of DEPT, COSY and HMQC experiments. Infrared spectra were recorded on a Sapphire-ATR Spectrophotometer; peaks are reported in $\mathrm{cm}^{-1}$. Optical rotation values were measured on an UV-vis Jasco J-175. High resolution mass spectra (HRMS) were recorded at Micromass-AutoSpec using (CI+). Qualitative enantiomeric enrichment of compound 11 was determined by CHPLC on a Waters 2690 chromatograph using a Daicel Chiracel OD 4.6 x $250 \mathrm{~mm}$ column $\left({ }^{\mathrm{i}} \mathrm{PrOH} / \mathrm{hexanes,} 1 / 9 ; 1 \mathrm{~mL} / \mathrm{min}\right)$.

The reactions concerning the preparation of compounds 16, 17, 18, and(-)-3 have been performed in a small scale (<25 mg) because all these compounds (with the unprotected amine functionality) present low stability. 


\section{1-[(1R)-1-(Hydroxymethyl)allyl]pyrrolidine-2,5-dione (11):}

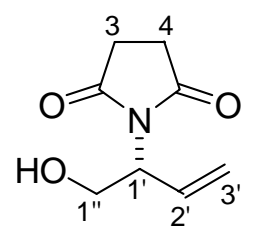

11

A mixture of $35 \mathrm{mg}(0.10 \mathrm{mmol})$ of $\pi$-allylpalladium chloride dimer, $218 \mathrm{mg}(0.28 \mathrm{mmol})$ of $(S, S)$ 19, $125 \mathrm{mg}(1.18 \mathrm{mmol})$ of sodium carbonate and $2.32 \mathrm{~g}(23.4 \mathrm{mmol})$ of succinimide (9) was purged with nitrogen for $1 \mathrm{~h}$. Dry $\mathrm{CH}_{2} \mathrm{Cl}_{2}(186 \mathrm{~mL})$ was added and the mixture was stirred at room temperature for $10 \mathrm{~min}$. Then, $1.9 \mathrm{~mL}(23.4 \mathrm{mmol})$ of butadiene monoepoxide $(\mathbf{1 0})$ were added and the resulting mixture was efficiently stirred under nitrogen for $14 \mathrm{~h}$. The solvent was removed under vacuum and the oily residue was purified by flash chromatography on silica gel (gradient hexanes:ethyl acetate 1:1 to ethyl acetate) to give $3.62 \mathrm{~g}(21.3 \mathrm{mmol}, 91 \%)$ of $\mathbf{1 1}$ as a colorless oil in $87 \%$ ee (determined by ${ }^{1} \mathrm{HNMR}$ analysis of its Mosher esters): $R_{\mathrm{f}}=0.30$ (ethyl acetate); $[\alpha]_{\mathrm{D}}{ }^{20}=$ +35 (c 0.95, $\left.\mathrm{CHCl}_{3}\right)$; IR (ATR): 3448, 2927, 1697, 1391, 1183; ${ }^{1} \mathbf{H}$ NMR (250 MHz, $\left.\mathrm{CDCl}_{3}\right): \delta$ $6.10\left(\mathrm{ddd}, J=17.1,10.5,7.3 \mathrm{~Hz}, 1 \mathrm{H}: \mathrm{H}_{2}\right), 5.25\left(\mathrm{~m}, 2 \mathrm{H}: 2 \mathrm{H}_{3}{ }^{\prime}\right), 4.79$ (m, $1 \mathrm{H}^{\prime} \mathrm{H}_{1}$ ) $), 4.06$ (dd, $J=11.5$, 8.0 Hz, 1H: $\mathrm{H}_{1}$ "), 3.85 (dd, $J=11.5,4.0 \mathrm{~Hz}, 1 \mathrm{H}: \mathrm{H}_{1}$ ") $), 2.75\left(\mathrm{~m}, 4 \mathrm{H}: 2 \mathrm{H}_{3}, 2 \mathrm{H}_{4}\right) ;{ }^{13} \mathbf{C ~ N M R}(62.5 \mathrm{MHz}$, $\left.\mathrm{CDCl}_{3}\right): \delta 177.8\left(\mathrm{C}_{2}, \mathrm{C}_{5}\right), 131.2\left(\mathrm{C}_{2}{ }^{\prime}\right), 119.1\left(\mathrm{C}_{3}{ }^{\prime}\right), 61.5\left(\mathrm{C}_{1}{ }^{\prime}\right), 56.2\left(\mathrm{C}_{1}{ }^{\prime}\right), 28.0\left(\mathrm{C}_{3}, \mathrm{C}_{4}\right)$. Anal. Calcd for $\mathrm{C}_{8} \mathrm{H}_{11} \mathrm{NO}_{3}$ : C, 56.80; H, 6.55; N, 8.28. Found: C, 56.81; H, 6.57; N, 8.11. For preparative purposes, the crude material can be used in the next step without chromatographic purification, with the overall yield being unaffected.

\section{1-[(1R)-1-(tert-Butyldiphenylsilyloxymethyl)allyl]pyrrolidine-2,5-dione (12):}




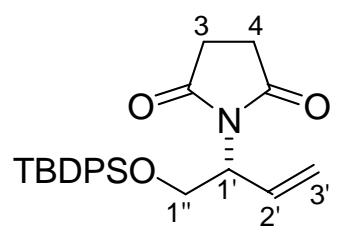

12

Imidazole (3.4 g, $50.4 \mathrm{mmol})$ and TBDPSCl $(5.5 \mathrm{~g}, 20.18 \mathrm{mmol})$ were added to a solution of 11 (1.7 $\mathrm{g}, 10.06 \mathrm{mmol})$ in dry $\mathrm{CH}_{2} \mathrm{Cl}_{2}(83 \mathrm{~mL})$ under nitrogen at $0{ }^{\circ} \mathrm{C}$. Then, the cooling bath was removed and the mixture stirred at room temperature, under nitrogen, for $14 \mathrm{~h}$. The solvent was evaporated under vacuum and replaced by ethyl acetate $(50 \mathrm{~mL})$. The resulting mixture was stirred vigorously and the insoluble fine white powder (imidazole $\cdot \mathrm{HCl})$ filtered trough celite. The filtrate was concentrated under vacuum and the resulting yellowish wax was purified by flash chromatography on silica gel (gradient, hexanes:ethyl acetate 9:1 to 7:3) to give 12 (3.89 g, 9.54 mmol, 95\%) of as a white solid in 87\% ee. Recrystallization from 2-propanol furnished 12 (3.67 g, $9.00 \mathrm{mmol}, 89 \%$ ) of $>98 \%$ ee: $R_{\mathrm{f}}=0.57$ (hexanes:ethyl acetate $\left.1: 1\right)$; mp $89-91{ }^{\circ} \mathrm{C}$ (2-propanol); $[\alpha]_{\mathrm{D}}{ }^{20}=-22\left(c 1.36, \mathrm{CHCl}_{3}\right)$; IR (ATR) 3069, 2928, 2858, 1702, 1390, 1359, 1195, 1111; ${ }^{1} \mathbf{H}$ NMR $\left(250 \mathrm{MHz}, \mathrm{CDCl}_{3}\right): \delta 7.75(\mathrm{~m}, 4 \mathrm{H}), 7.40(\mathrm{~m}, 6 \mathrm{H}), 6.04\left(\mathrm{ddd}, J=17.4,10.4,7.9 \mathrm{~Hz}, 1 \mathrm{H}: \mathrm{H}_{2},\right), 5.20$

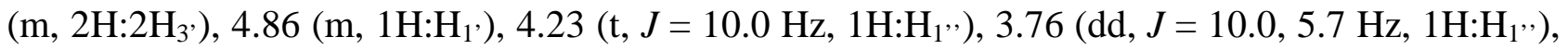
$2.64\left(\mathrm{~s}, 4 \mathrm{H}: 2 \mathrm{H}_{3}, 2 \mathrm{H}_{4}\right), 1.00(\mathrm{~s}, 9 \mathrm{H}) ;{ }^{13} \mathrm{C} \mathrm{NMR}\left(62.5 \mathrm{MHz}, \mathrm{CDCl}_{3}\right): \delta 176.9\left(\mathrm{C}_{2}, \mathrm{C}_{5}\right), 135.5\left(\mathrm{C}_{\mathrm{Ph}}\right)$, 135.4 $\left(\mathrm{C}_{\mathrm{Ph}}\right), 133.2\left(\mathrm{C}_{\mathrm{Ph}}\right), 133.0\left(\mathrm{C}_{\mathrm{Ph}}\right), 131.3\left(\mathrm{C}_{2}\right), 129.8\left(\mathrm{C}_{\mathrm{Ph}}\right), 129.7\left(\mathrm{C}_{\mathrm{Ph}}\right), 127.7\left(\mathrm{C}_{\mathrm{Ph}}\right), 119.5\left(\mathrm{C}_{3^{\prime}}\right)$, $62.1\left(\mathrm{C}_{1}{ }^{\prime}\right), 56.3\left(\mathrm{C}_{1}{ }^{\prime}\right), 28.0\left(\mathrm{C}_{3}, \mathrm{C}_{4}\right), 26.6,19.0 ; \mathrm{MS}(\mathrm{ESI}, \mathrm{MeOH}) \mathrm{m} / z 430\left(\mathrm{MNa}^{+}\right)$. Anal. Calcd for $\mathrm{C}_{24} \mathrm{H}_{29} \mathrm{NO}_{3} \mathrm{Si}: \mathrm{C}, 70.72 ; \mathrm{H}, 7.17 ; \mathrm{N}, 3.44$. Found: C, 71.02; H, 7.20; N, 3.26.

The ee of 12 was determined by desilylation with $\mathrm{Et}_{3} \mathrm{~N} \cdot 3 \mathrm{HF}$ in $\mathrm{THF}$ to give 11, followed by ${ }^{1} \mathrm{HNMR}$ analysis of the corresponding Mosher esters. 
(5RS)-1-[(1R)-1-(tert-Butyldiphenylsilyloxymethyl)allyl]-5-hydroxypyrrolidin-2-one (13):

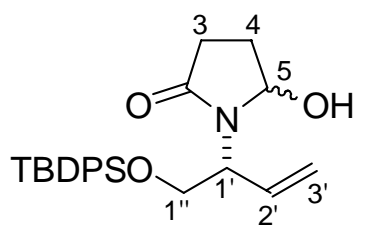

13

A solution of $\mathrm{LiBEt}_{3} \mathrm{H}$ in $\mathrm{THF}(1 \mathrm{M}, 1.1 \mathrm{~mL}, 1.1 \mathrm{mmol})$ was added dropwise to a solution of 12 (272.0 $\mathrm{mg}, 0.67 \mathrm{mmol})$ in dry $\mathrm{THF}(2.5 \mathrm{~mL})$ at $-78^{\circ} \mathrm{C}$ and the reaction mixture was stirred at the same temperature for exactly $45 \mathrm{~min}$. Keeping the temperature at $-78{ }^{\circ} \mathrm{C}$, saturated aqueous $\mathrm{NaHCO}_{3}(5 \mathrm{~mL})$ and $\mathrm{H}_{2} \mathrm{O}_{2}(1.2 \mathrm{~mL})$ were added, the mixture was let to warm up slowly to room temperature and then it was stirred for one additional hour. After filtration trough celite, the solution was extracted with $\mathrm{CH}_{2} \mathrm{Cl}_{2}(4 \times 4 \mathrm{~mL})$, the combined organic extracts were dried over anhydrous $\mathrm{Na}_{2} \mathrm{SO}_{4}$ and concentrated under vacuum. The oily residue was purified by flash chromatography on silica gel (gradient, hexanes to hexanes:ethyl acetate 7:3) to give a mixture of isomers $\mathbf{1 3}$ (239 mg, $0.58 \mathrm{mmol}, 87 \%)$ as a colorless wax: $[\alpha]_{\mathrm{D}}{ }^{20}=+13\left(c\right.$ 1.20, $\left.\mathrm{CH}_{2} \mathrm{Cl}_{2}\right)$; IR (ATR): 3301, 3073, 2930, 2855, 1664, 1422, 1109; ${ }^{1} \mathbf{H}$ NMR (250 MHz, $\left.\mathrm{CDCl}_{3}\right)$ : isomers A (major) and B (minor) $\delta 7.65$ (m, $4 \mathrm{H}_{\mathrm{A}}$ and $\left.4 \mathrm{H}_{\mathrm{B}}\right), 7.38\left(\mathrm{~m}, 6 \mathrm{H}_{\mathrm{A}}\right.$ and $\left.6 \mathrm{H}_{\mathrm{B}}\right), 6.04\left(\mathrm{ddd}, J=17.4,10.4,7.0 \mathrm{~Hz}, 1 \mathrm{H}: \mathrm{H}_{2}{ }^{\prime} \mathrm{B}\right), 5.80(\mathrm{ddd}, J=$ 17.2, 10.6, $\left.6.1 \mathrm{~Hz}, 1 \mathrm{H}^{\prime} \mathrm{H}_{2}{ }^{\prime} \mathrm{A}\right), 5.42\left(\mathrm{dt}, J=5.2,1.8,1 \mathrm{H}: \mathrm{H}_{5 \mathrm{~A}}\right), 5.38\left(\mathrm{~m}, 1 \mathrm{H}: \mathrm{H}_{5 \mathrm{~B}}\right), 5.20\left(\mathrm{~m}, 2 \mathrm{H}^{\prime} 2 \mathrm{H}_{3^{\prime} \mathrm{B}}\right)$, $5.20\left(\mathrm{dt}, J=10.6,1.3 \mathrm{~Hz}, 1 \mathrm{H}: \mathrm{H}_{3}{ }^{\prime} \mathrm{A}\right), 5.10\left(\mathrm{dt}, J=17.2,1.3 \mathrm{~Hz}, 1 \mathrm{H}: \mathrm{H}_{3^{\prime}} \mathrm{A}\right), 4.86(\mathrm{~m}, 1 \mathrm{H}: \mathrm{OH}), 4.74(\mathrm{~m}$, $\left.1 \mathrm{H}: \mathrm{H}_{1}{ }^{\prime} \mathrm{A}\right), 4.37$ (m, 1H: $\left.\mathrm{H}_{1}{ }^{\prime} \mathrm{B}\right), 4.09$ (dd, $\left.J=10.7,8.9 \mathrm{~Hz}, 1 \mathrm{H}: \mathrm{H}_{1}{ }^{\prime \prime} \mathrm{B}\right), 3.92(\mathrm{dd}, J=11.1,3.1 \mathrm{~Hz}$, 1H: $\left.\mathrm{H}_{1}{ }^{\prime}{ }^{A}\right), 3.80\left(\mathrm{dd}, J=11.1,4.7 \mathrm{~Hz}, 1 \mathrm{H}: \mathrm{H}_{1}{ }^{\prime}{ }^{\prime}\right), 3.78$ (dd, $\left.J=10.7,3.9 \mathrm{~Hz}, 1 \mathrm{H}: \mathrm{H}_{1}{ }^{\prime}{ }^{\prime}\right), 2.74(\mathrm{dt}, J=$ 16.8, $\left.9.3 \mathrm{~Hz}, 1 \mathrm{H}: \mathrm{H}_{3 \mathrm{~A}}\right), 2.63\left(\mathrm{~m}, 1 \mathrm{H}_{\mathrm{B}}\right), 2.35\left(\mathrm{ddd}, J=16.8,8.8,2.6 \mathrm{~Hz}, 1 \mathrm{H}: \mathrm{H}_{3 \mathrm{~A}}\right), 2.14(\mathrm{~m}$, $\left.2 \mathrm{H}_{\mathrm{A}}+3 \mathrm{H}_{\mathrm{B}}: 2 \mathrm{H}_{4 \mathrm{~A}}, 3 \mathrm{H}_{\mathrm{B}}\right), 1.08\left(\mathrm{~s}, 9 \mathrm{H}_{\mathrm{A}}\right), 1.06\left(\mathrm{~s}, 9 \mathrm{H}_{\mathrm{B}}\right) ;{ }^{13} \mathbf{C} \mathbf{N M R}\left(62.5 \mathrm{MHz}, \mathrm{CDCl}_{3}\right)$ : isomer A $\delta 175.5$ $\left(\mathrm{C}_{2}\right), 135.8\left(\mathrm{C}_{\mathrm{Ph}}\right), 135.5\left(\mathrm{C}_{\mathrm{Ph}}\right), 132.5\left(\mathrm{C}_{\mathrm{Ph}}\right), 131.8\left(\mathrm{C}_{2},\right), 130.2\left(\mathrm{C}_{\mathrm{Ph}}\right), 128.0\left(\mathrm{C}_{\mathrm{Ph}}\right), 127.9\left(\mathrm{C}_{\mathrm{Ph}}\right), 118.5$ 
$\left(\mathrm{C}_{3^{\prime}}\right), 80.5\left(\mathrm{C}_{5}\right), 65.5\left(\mathrm{C}_{1^{\prime}}\right), 54.5\left(\mathrm{C}_{1^{\prime}}\right), 28.8 / 27.7\left(\mathrm{C}_{3} / \mathrm{C}_{4}\right), 26.9,19.2$; HRMS (CI+): calcd for $\mathrm{C}_{24} \mathrm{H}_{32} \mathrm{NO}_{3} \mathrm{Si}: 410.2151[\mathrm{M}+\mathrm{H}]^{+}$; found: 410.2150 .

(5R)-1-[(1R)-1-(tert-Butyldiphenylsilyloxymethyl)allyl]-5-[(2R)-5-oxo-3-vinyl-2,5-dihydro-2furyl]pyrrolidin-2-one (14a):

(norsecurinine numbering)

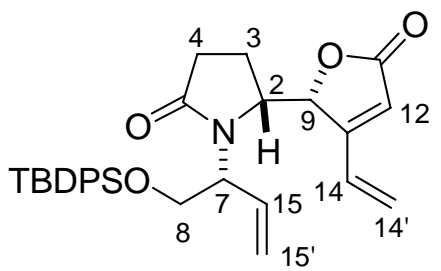

$14 a$

In a $250 \mathrm{~mL}$ schlenk vessel connected to a nitrogen line, aminal $\mathbf{1 3}(2.70 \mathrm{~g}, 6.6 \mathrm{mmol})$ and silyloxyfurane 8 (2.20 g, $8.26 \mathrm{mmol})$ were dissolved in dry diethyl ether $(130 \mathrm{~mL})$. The solution was cooled down to $0{ }^{\circ} \mathrm{C}, \mathrm{BF}_{3} \cdot \mathrm{Et}_{2} \mathrm{O}(2.1 \mathrm{~mL}, 16.37 \mathrm{mmol})$ was slowly added and the reaction mixture was kept at the same temperature for $1.5 \mathrm{~h}$. Then, it was treated with saturated aqueous $\mathrm{NaHCO}_{3}(100 \mathrm{~mL})$, the aqueous phase was extracted with $\mathrm{CH}_{2} \mathrm{Cl}_{2}(3 \times 100 \mathrm{~mL})$, the combined organic extracts were dried over anhydrous $\mathrm{Na}_{2} \mathrm{SO}_{4}$ and concentrated in vacuo. The colorless oily residue was let to stand at room temperature for $24 \mathrm{~h}$. A solid was formed, which was separated by filtration and washed with cold diethyl ether, affording $14 \mathrm{a}(1.70 \mathrm{~g}, 3.39 \mathrm{mmol}, 51 \%)$ as colorless crystals. The filtrate was concentrated under vacuum and the residue identified as a mixture of isomers 14a-d by ${ }^{1} \mathrm{H}$ NMR analysis. 14a: $R_{\mathrm{f}}=0.28$ (hexanes:ethyl acetate $\left.1: 1\right) ;[\alpha]_{\mathrm{D}}{ }^{20}=+252(c$ 1.00, $\left.\mathrm{CH}_{2} \mathrm{Cl}_{2}\right)$; IR (ATR): 3075, 2931, 2858, 1758, 1687, 1420, 1109; ${ }^{1} \mathbf{H} \mathbf{~ N M R}\left(250 \mathrm{MHz}, \mathrm{CDCl}_{3}\right)$ : $\delta 7.60(\mathrm{~m}, 4 \mathrm{H}), 7.40(\mathrm{~m}, 6 \mathrm{H}), 6.57\left(\mathrm{dd}, J=17.8,11.0 \mathrm{~Hz}, 1 \mathrm{H}: \mathrm{H}_{14}\right), 6.08(\mathrm{ddd}, J=17.6,10.5,7.2$ $\left.\mathrm{Hz}, 1 \mathrm{H}: \mathrm{H}_{15}\right), 6.01\left(\mathrm{~s}, 1 \mathrm{H}: \mathrm{H}_{12}\right), 5.76\left(\mathrm{~d}, J=17.8 \mathrm{~Hz}, 1 \mathrm{H}: \mathrm{H}_{14^{\prime}}\right), 5.66\left(\mathrm{~d}, J=11.0 \mathrm{~Hz}, 1 \mathrm{H}: \mathrm{H}_{14^{\prime}}\right), 5.17(\mathrm{br}$ 
s, $\left.1 \mathrm{H}: \mathrm{H}_{9}\right), 5.06$ (d, $J=10.5 \mathrm{~Hz}, 1 \mathrm{H}: \mathrm{H}_{15}$ ), 4.91 (d, $J=17.6 \mathrm{~Hz}, 1 \mathrm{H}: \mathrm{H}_{15}$ ), 4.29 (m, 2H: $\left.\mathrm{H}_{2}, \mathrm{H}_{8}\right), 3.60$ (m, 2H: $\left.\mathrm{H}_{7}, \mathrm{H}_{8}\right), 2.56\left(\mathrm{~m}, 1 \mathrm{H}: \mathrm{H}_{4}\right), 2.24\left(\mathrm{~m}, 2 \mathrm{H}: \mathrm{H}_{3}, \mathrm{H}_{4}\right), 1.98\left(\mathrm{~m}, 1 \mathrm{H}: \mathrm{H}_{3}\right), 1.03(\mathrm{~s}, 9 \mathrm{H}) ;{ }^{13} \mathbf{C ~ N M R}(62.5$ $\left.\mathrm{MHz}, \mathrm{CDCl}_{3}\right): \delta 176.3\left(\mathrm{C}_{5}\right), 171.4\left(\mathrm{C}_{11}\right), 161.1\left(\mathrm{C}_{13}\right), 135.45\left(\mathrm{C}_{\mathrm{Ph}}\right), 135.42\left(\mathrm{C}_{\mathrm{Ph}}\right), 133.5\left(\mathrm{C}_{\mathrm{Ph}}\right), 133.2$ $\left(\mathrm{C}_{\mathrm{Ph}}\right), 132.9\left(\mathrm{C}_{15}\right), 129.9\left(\mathrm{C}_{\mathrm{Ph}}\right), 127.8\left(\mathrm{C}_{\mathrm{Ph}}\right), 127.5\left(\mathrm{C}_{14}\right), 124.3\left(\mathrm{C}_{14}\right), 119.0\left(\mathrm{C}_{12}\right), 117.7\left(\mathrm{C}_{15}\right), 83.4$ $\left(\mathrm{C}_{9}\right), 64.1\left(\mathrm{C}_{8}\right), 62.6\left(\mathrm{C}_{7}\right), 62.0\left(\mathrm{C}_{2}\right), 30.9\left(\mathrm{C}_{4}\right), 26.8(\mathrm{Me}), 23.1\left(\mathrm{C}_{3}\right), 19.1\left(\mathrm{CMe}_{3}\right)$; HRMS $(\mathrm{CI}+)$ : calcd for $\mathrm{C}_{30} \mathrm{H}_{36} \mathrm{NO}_{4} \mathrm{Si}: 502.2414[\mathrm{M}+\mathrm{H}]^{+}$; found: 502.2417 .

$(6 R, 10 \mathrm{a} R, 10 \mathrm{~b} R)-6$-tert-Butyldiphenylsilyloxymethyl-9,10,10a,10b-tetrahydro-6H-furo[2,3$c]$ pyrrolo[1,2-a]azepine-2,8-dione (15a):

(norsecurinine numbering)

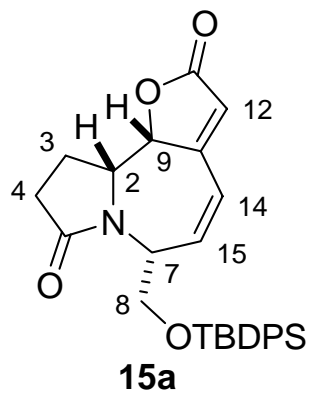

A solution of second generation Grubbs catalyst (17 mg, $0.02 \mathrm{mmol}$ ) in degassed anhydrous $\mathrm{CH}_{2} \mathrm{Cl}_{2}$ (7 mL) was slowly added to a solution of crystalline $\mathbf{1 4 a}\left(105 \mathrm{mg}, 0.21 \mathrm{mmol}\right.$ ) in degassed $\mathrm{CH}_{2} \mathrm{Cl}_{2}$ $(21 \mathrm{~mL})$ under argon and the reaction mixture was stirred for $14 \mathrm{~h}$ at room temperature. Then, the solvent was evaporated under vacuum and the dark brown oily residue was purified by flash chromatography (gradient, hexanes to hexanes:ethyl acetate 1:1) to furnish compound 15a (99 mg, $0.21 \mathrm{mmol}, 100 \%)$ as a pale brownish oil: $\mathrm{R}_{f}=0.41$ (hexanes:ethyl acetate $\left.1: 1\right) ;[\alpha]_{\mathrm{D}}{ }^{20}=+118(c$ 0.61, $\mathrm{CHCl}_{3}$ ); IR (ATR): 3070, 2928, 2854, 1752, 1688, 1421, 1109; ${ }^{\mathbf{H}} \mathbf{H} \mathbf{N M R}\left(250 \mathrm{MHz}, \mathrm{CDCl}_{3}\right)$ : $\delta 7.65(\mathrm{~m}, 4 \mathrm{H}), 7.40(\mathrm{~m}, 6 \mathrm{H}), 6.65\left(\mathrm{dd}, J=10.4,3.4 \mathrm{~Hz}, 1 \mathrm{H}: \mathrm{H}_{15}\right), 6.49(\mathrm{dd}, J=10.4,2.5 \mathrm{~Hz}$, 
1H:H $\mathrm{H}_{14}$ ), 6.01 (br s, 1H:H $\mathrm{H}_{12}$ ), 5.38 (br d, $\left.J=7.5 \mathrm{~Hz}, 1 \mathrm{H}: \mathrm{H}_{9}\right) .4 .58$ (dd, $\left.J=10.2,8.8 \mathrm{~Hz}, 1 \mathrm{H}: \mathrm{H}_{8}\right), 4.43$ $\left(\mathrm{q}, J=7.5 \mathrm{~Hz}, 1 \mathrm{H}: \mathrm{H}_{2}\right), 4.23\left(\mathrm{dd}, J=10.2,6.0 \mathrm{~Hz}, 1 \mathrm{H}: \mathrm{H}_{8}\right), 3.82\left(\mathrm{~m}, 1 \mathrm{H} \mathrm{H}_{7}\right), 2.31(\mathrm{dt}, J=16.6,10.3$ $\left.\mathrm{Hz}, 1 \mathrm{H}: \mathrm{H}_{4}\right), 2.01\left(\mathrm{~m}, 2 \mathrm{H}: \mathrm{H}_{3}, \mathrm{H}_{4}\right), 1.44\left(\mathrm{~m}, 1 \mathrm{H}: \mathrm{H}_{3}\right), 1.07(\mathrm{~s}, 9 \mathrm{H}) ;{ }^{13} \mathbf{C} \mathbf{N M R}\left(62.5 \mathrm{MHz}, \mathrm{CDCl}_{3}\right): \delta$ $175.0\left(\mathrm{C}_{5}\right), 172.2\left(\mathrm{C}_{11}\right), 161.0\left(\mathrm{C}_{13}\right), 145.5\left(\mathrm{C}_{15}\right), 135.6\left(\mathrm{C}_{\mathrm{Ph}}\right), 135.5\left(\mathrm{C}_{\mathrm{Ph}}\right), 133.4\left(\mathrm{C}_{\mathrm{Ph}}\right), 133.1\left(\mathrm{C}_{\mathrm{Ph}}\right)$, $130.0\left(\mathrm{C}_{\mathrm{Ph}}\right), 127.8\left(\mathrm{C}_{\mathrm{Ph}}\right), 123.0\left(\mathrm{C}_{14}\right), 118.1\left(\mathrm{C}_{12}\right), 81.9\left(\mathrm{C}_{9}\right), 63.2\left(\mathrm{C}_{8}\right), 58.9\left(\mathrm{C}_{2}\right), 56.2\left(\mathrm{C}_{7}\right)$, 30.2/29.6 $\left(\mathrm{C}_{3} / \mathrm{C}_{4}\right), 26.8(\mathrm{Me}), 19.1$ and $19.0\left(C \mathrm{Me}_{3}\right)$; HRMS (CI+): calcd for $\mathrm{C}_{28} \mathrm{H}_{32} \mathrm{NO}_{4} \mathrm{Si}$ : 474.2101 $[\mathrm{M}+\mathrm{H}]^{+}$; found: 474.2111 .

This reaction has been performed with quantities of $\mathbf{1 4 a}$ up to $700 \mathbf{m g}$. Yields of different runs are in the range $80 \%$ to $>98 \%$.

$(6 R, 10 \mathrm{a} R, 10 \mathrm{~b} R)-6$-(tert-Butyldiphenylsilyloxymethyl)-9,10,10a,10b-tetrahydro-6H-furo[2,3c]pyrrolo[1,2-a ]azepin-2(8H)-one (16):

(norsecurinine numbering)

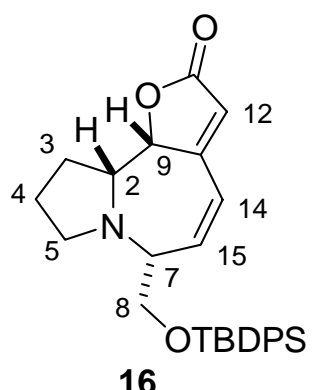

A solution of $\mathrm{AlH}_{3}$ in $\mathrm{THF}^{1}(1.0 \mathrm{M}, 84 \mu \mathrm{L}, 0.084 \mathrm{mmol})$ was added dropwise to a stirred solution of 15a $(22 \mathrm{mg}, 0.05 \mathrm{mmol})$ in dry $\operatorname{THF}(75 \mu \mathrm{L})$ at $0{ }^{\circ} \mathrm{C}$ under nitrogen. The reaction mixture was kept at the same temperature for strictly $5 \mathrm{~min},{ }^{2} \mathrm{MeOH}(0.5 \mathrm{~mL})$ was added, the resulting mixture was let to warm up to room temperature and then filtered trough a pad of silica gel $(15 \times 15 \mathrm{~mm})$. The solvent was removed under vacuum and the oily residue was purified by flash chromatography on 
silica gel (gradient, hexanes to hexanes:ethyl acetate 7:1) to furnish $16(11 \mathrm{mg}, 0.02 \mathrm{mmol}, 57 \%)$ as a yellow oil: $R_{\mathrm{f}}=0.64$ (hexanes:ethyl acetate $\left.1: 1\right) ;[\alpha]_{\mathrm{D}}{ }^{20}=+89\left(c 1.39, \mathrm{CHCl}_{3}\right) ;$ IR $(\mathrm{ATR}): 3072$, 2930, 2858, 1754, 1110; ${ }^{1} \mathbf{H}$ NMR $\left(250 \mathrm{MHz}, \mathrm{CDCl}_{3}\right): \delta 7.65$ (m, 4H), $7.40(\mathrm{~m}, 6 \mathrm{H}), 6.66$ (br d, $J$ $\left.=10.8 \mathrm{~Hz}, 1 \mathrm{H}: \mathrm{H}_{14} / \mathrm{H}_{15}\right), 6.49$ (ddd, $\left.J=10.8,3.0,1.4 \mathrm{~Hz}, 1 \mathrm{H}: \mathrm{H}_{14} / \mathrm{H}_{15}\right), 5.93$ (d, $\left.J=1.4 \mathrm{~Hz}, 1 \mathrm{H}: \mathrm{H}_{12}\right)$, $5.27\left(\mathrm{dd}, J=6.4,1.4 \mathrm{~Hz}, 1 \mathrm{H}: \mathrm{H}_{9}\right) .4 .05\left(\mathrm{q}, J=7.5 \mathrm{~Hz}, 1 \mathrm{H}: \mathrm{H}_{2}\right), 3.80\left(\mathrm{~m}, 3 \mathrm{H}: \mathrm{H}_{7}, 2 \mathrm{H}_{8}\right), 2.57(\mathrm{~m}$, $\left.1 \mathrm{H}: \mathrm{H}_{5}\right), 2.34\left(\mathrm{~m}, 1 \mathrm{H}: \mathrm{H}_{5}\right), 1.80(\mathrm{~m}, 1 \mathrm{H}), 1.59(\mathrm{~m}, 2 \mathrm{H}), 1.14(\mathrm{~m}, 1 \mathrm{H}), 1.07(\mathrm{~s}, 9 \mathrm{H}) ;{ }^{13} \mathbf{C ~ N M R}(62.5$ $\left.\mathrm{MHz}, \mathrm{CDCl}_{3}\right): \delta 173.4\left(\mathrm{C}_{11}\right), 163.3\left(\mathrm{C}_{13}\right), 146.2\left(\mathrm{C}_{15}\right), 135.6\left(\mathrm{C}_{\mathrm{Ph}}\right), 133.4\left(\mathrm{C}_{\mathrm{Ph}}\right), 129.9\left(\mathrm{C}_{\mathrm{Ph}}\right), 127.8$ $\left(\mathrm{C}_{\mathrm{Ph}}\right), 123.1\left(\mathrm{C}_{14}\right), 116.1\left(\mathrm{C}_{12}\right), 84.2\left(\mathrm{C}_{9}\right), 65.9\left(\mathrm{C}_{8}\right), 60.9 / 58.7\left(\mathrm{C}_{2} / \mathrm{C}_{7}\right), 47.4\left(\mathrm{C}_{5}\right), 26.9(\mathrm{Me})$, 24.6/24.1 $\left(\mathrm{C}_{3} / \mathrm{C}_{4}\right), 19.2\left(\mathrm{CMe}_{3}\right)$; HRMS $(\mathrm{CI}+)$ : calcd for $\mathrm{C}_{28} \mathrm{H}_{34} \mathrm{NO}_{3} \mathrm{Si}$ : $460.2308[\mathrm{M}+\mathrm{H}]^{+}$; found: 460.2292 .

${ }^{1}$ Giraud, L.; Huber, V.; Jenny, T. Tetrahedron 1998, 54, 11899-11906.

${ }^{2}$ A $10 \%$ of the starting lactam was recovered unchanged, but longer reaction times furnished over-reduction products. The use of lower molar amounts of aluminum hydride resulted in low conversion of 15a.

$(6 R, 10 \mathrm{a} R, 10 \mathrm{~b} R)-6-H y d r o x y m e t h y l-9,10,10 \mathrm{a}, 10 \mathrm{~b}-t e t r a h y d r o-6 H$-furo[2,3-c]pyrrolo[1,2a]azepin-2(8H)-one (17):

(norsecurinine numbering)

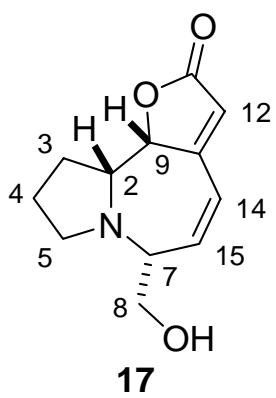

A solution of $16(22 \mathrm{mg}, 0.05 \mathrm{mmol})$ in THF $(640 \mu \mathrm{L})$, at room temperature, was treated with $\mathrm{Et}_{3} \mathrm{~N} \cdot 3 \mathrm{HF}(47 \mu \mathrm{L}, 0.29 \mathrm{mmol})$. The reaction mixture was stirred for $7 \mathrm{~h}$ at the same temperature. 
Then, $\mathrm{CH}_{2} \mathrm{Cl}_{2}(1 \mathrm{~mL})$ and saturated aqueous $\mathrm{NaHCO}_{3}(1 \mathrm{~mL})$ were added. The layers were separated and the aqueous phase was extracted with $\mathrm{CH}_{2} \mathrm{Cl}_{2}(3 \times 0.5 \mathrm{~mL})$. The combined organic extracts were dried over anhydrous $\mathrm{MgSO}_{4}$ and concentrated under vacuum and the oily residue was purified by flash chromatography on silica gel (gradient, $\mathrm{CH}_{2} \mathrm{Cl}_{2}$ to $\mathrm{CH}_{2} \mathrm{Cl}_{2}: \mathrm{MeOH} 19: 1$ ) to furnish $\mathbf{1 7}^{1}(10.0 \mathrm{mg}, 0.04 \mathrm{mmol}, 94 \%)$ as a colorless oil: $R_{\mathrm{f}}=0.37\left(\mathrm{CH}_{2} \mathrm{Cl}_{2}:\right.$ acetone $\left.7: 3\right) ;[\alpha]_{\mathrm{D}}{ }^{20}=$ $+123\left(c 0.60, \mathrm{CH}_{2} \mathrm{Cl}_{2}\right) ;{ }^{1} \mathbf{H}$ NMR $\left(250 \mathrm{MHz}, \mathrm{CDCl}_{3}\right): \delta 6.69\left(\mathrm{dd}, J=10.7,2.5 \mathrm{~Hz}, 1 \mathrm{H}: \mathrm{H}_{14}\right), 6.02$ (br s, $\left.1 \mathrm{H}: \mathrm{H}_{12}\right), 5.95\left(\mathrm{dd}, J=10.7,3.9 \mathrm{~Hz}, 1 \mathrm{H}: \mathrm{H}_{15}\right), 5.40\left(\mathrm{~d}, J=7.4 \mathrm{~Hz}, 1 \mathrm{H}: \mathrm{H}_{9}\right), 4.20(\mathrm{q}, J \approx 7.6 \mathrm{~Hz}$, 1H: $\left.\mathrm{H}_{2}\right), 3.97\left(\mathrm{~m}, 1 \mathrm{H}: \mathrm{H}_{7}\right), 3.68\left(\mathrm{dd}, J=10.6,5.9 \mathrm{~Hz}, 1 \mathrm{H}: \mathrm{H}_{8}\right), 3.57\left(\mathrm{t}, J=10.6 \mathrm{~Hz}, 1 \mathrm{H}: \mathrm{H}_{8}\right), 2.74(\mathrm{~m}$,

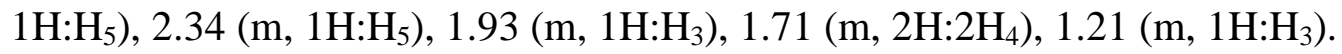

$(6 R, 10 \mathrm{a} R, 10 \mathrm{~b} R)-2-O x 0-2,8,9,10,10 \mathrm{a}, 10 \mathrm{~b}-h e x a h y d r o-6 H$-furo[2,3-c]pyrrolo[1,2-a]azepin-6yl]methyl methanesulfonate (18):

(norsecurinine numbering)

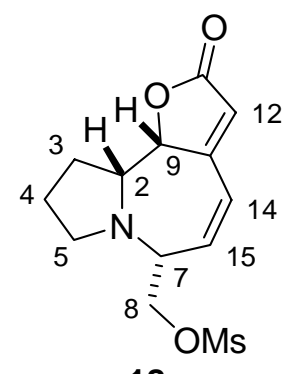

18

A solution of compound $\mathbf{1 7}(13 \mathrm{mg}, 0.06 \mathrm{mmol})$ in anhydrous $\mathrm{CH}_{2} \mathrm{Cl}_{2}(2.2 \mathrm{~mL})$ under nitrogen was cooled down to $0{ }^{\circ} \mathrm{C}, \mathrm{Et}_{3} \mathrm{~N}(37 \mu \mathrm{L}, 0.26 \mathrm{mmol})$ and $\mathrm{MsCl}(15 \mu \mathrm{L}, 0.19 \mathrm{mmol})$ were successively added, and the mixture was stirred at the same temperature for $30 \mathrm{~min}$. Then, saturated aqueous

\footnotetext{
${ }^{1}$ P. A. Jacobi, C. A. Blum, R. W. DeSimone, U. E. S. Udodong, J. Am. Chem. Soc. 1991, 113, 5384-5392.
} 
$\mathrm{NaHCO}_{3}(1 \mathrm{~mL})$ was added, the layers were separated and the aqueous phase was extracted with $\mathrm{CH}_{2} \mathrm{Cl}_{2}(4 \times 7 \mathrm{~mL})$. The combined organic extracts were dried over anhydrous $\mathrm{MgSO}_{4}$ and concentrated under vacuum and the oily residue was purified by flash chromatography on silica gel $\left(\mathrm{CH}_{2} \mathrm{Cl}_{2}: \mathrm{MeOH} 19: 1\right)$, affording $\mathbf{1 8}^{1}(17.0 \mathrm{mg}, 0.06 \mathrm{mmol}, 97 \%)$ of as pale yellow oil: $R_{\mathrm{f}}=0.79$ (acetone: $\left.\mathrm{CH}_{2} \mathrm{Cl}_{2} 3: 7\right) ;[\alpha]_{\mathrm{D}}{ }^{20}=+259\left(c 0.37, \mathrm{CH}_{2} \mathrm{Cl}_{2}\right) ;{ }^{1} \mathbf{H} \mathbf{N M R}\left(250 \mathrm{MHz}, \mathrm{CDCl}_{3}\right): \delta 6.73(\mathrm{dd}, J$ $\left.=10.8,2.5 \mathrm{~Hz}, 1 \mathrm{H}: \mathrm{H}_{14}\right), 6.24\left(\mathrm{dd}, J=10.8,3.9 \mathrm{~Hz}, 1 \mathrm{H}: \mathrm{H}_{15}\right), 6.01$ (br s, $\left.1 \mathrm{H}: \mathrm{H}_{12}\right), 5.37(\mathrm{~d}, J=7.2$ $\left.\mathrm{Hz}, 1 \mathrm{H}: \mathrm{H}_{9}\right), 4.42\left(\mathrm{dd}, J=10.0,6.8 \mathrm{~Hz}, 1 \mathrm{H}: \mathrm{H}_{8}\right), 4.29\left(\mathrm{dd}, J=10.0,7.3 \mathrm{~Hz}, 1 \mathrm{H}: \mathrm{H}_{8}\right), 4.15(\mathrm{~m}$,

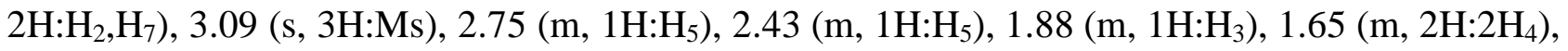
$1.21\left(\mathrm{~m}, 1 \mathrm{H}: \mathrm{H}_{3}\right)$.

\section{(-)-Norsecurinine, (-)-3:}

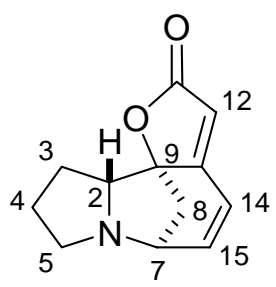

$(-)-3$

A solution of KHMDS in toluene $(0.5 \mathrm{M}, 56 \mu \mathrm{L}, 0.03 \mathrm{mmol})$ was added to a schlenk tube, under nitrogen, containing a solution of compound $18(7.0 \mathrm{mg}, 0.02 \mathrm{mmol})$ in $\operatorname{dry} \mathrm{CH}_{2} \mathrm{Cl}_{2}(4 \mathrm{~mL})$ at -78 ${ }^{\circ} \mathrm{C}$. After stirring for $15 \mathrm{~min}$, the mixture was warmed up to room temperature and additionally stirred for $30 \mathrm{~min}$. Then, the reaction mixture was cooled down again to $-78{ }^{\circ} \mathrm{C}$ and cold $\left(0{ }^{\circ} \mathrm{C}\right)$ saturated aqueous $\mathrm{NaHCO}_{3}(5 \mathrm{~mL})$ was added. After warming at room temperature, the organic phase was decanted and the aqueous layer was extracted with $\mathrm{CH}_{2} \mathrm{Cl}_{2}(3 \times 8 \mathrm{~mL})$. The combined organic extracts were dried over anhydrous $\mathrm{MgSO}_{4}$, the solvent was removed under vacuum and the remaining oily material was purified by flash chromatography on silica gel $\left(\mathrm{CH}_{2} \mathrm{Cl}_{2}\right.$ : $\left.\mathrm{MeOH} 19: 1\right)$ 
to furnish (-)-3 (4.0 mg, $0.02 \mathrm{mmol}, 80 \%)$ as a colorless oil: $R_{\mathrm{f}}=0.30$ (ethyl acetate); $[\alpha]_{\mathrm{D}}{ }^{20}=-270$ $\left(c\right.$ 0.20, EtOH); ${ }^{1} \mathbf{H}$ NMR $\left(400 \mathrm{MHz}, \mathrm{CDCl}_{3}\right): \delta 6.75\left(\mathrm{dd}, J=9.1,6.5 \mathrm{~Hz}, 1 \mathrm{H}: \mathrm{H}_{15}\right), 6.49(\mathrm{~d}, J=9.1$

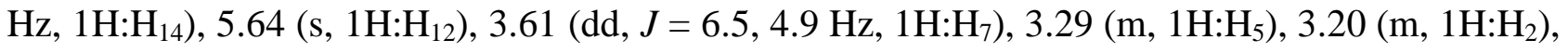
$2.56\left(\mathrm{dd}, J=10.8,4.9 \mathrm{~Hz}, 1 \mathrm{H}: \mathrm{H}_{8}\right), 2.50\left(\mathrm{~m}, 1 \mathrm{H}: \mathrm{H}_{5}\right), 1.98\left(\mathrm{~m}, 2 \mathrm{H}: 2 \mathrm{H}_{3}\right), 1.79\left(\mathrm{~m}, 2 \mathrm{H}: 2 \mathrm{H}_{4}\right), 1.72(\mathrm{~d}, J$ $\left.=10.8 \mathrm{~Hz}, 1 \mathrm{H}: \mathrm{H}_{8}\right)$. 


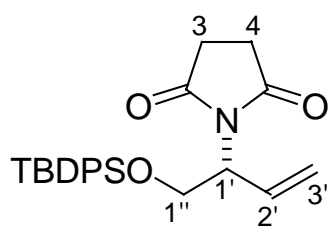

12

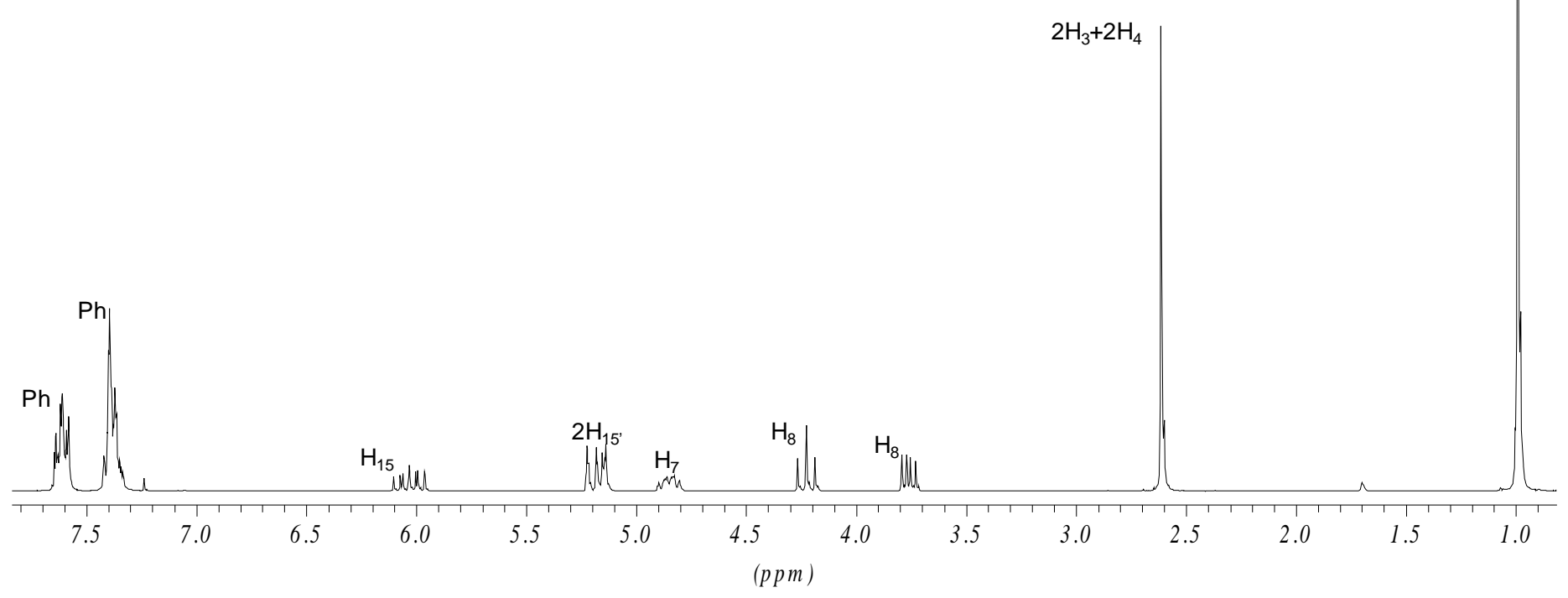




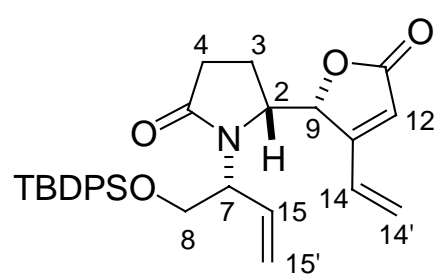

$14 a$

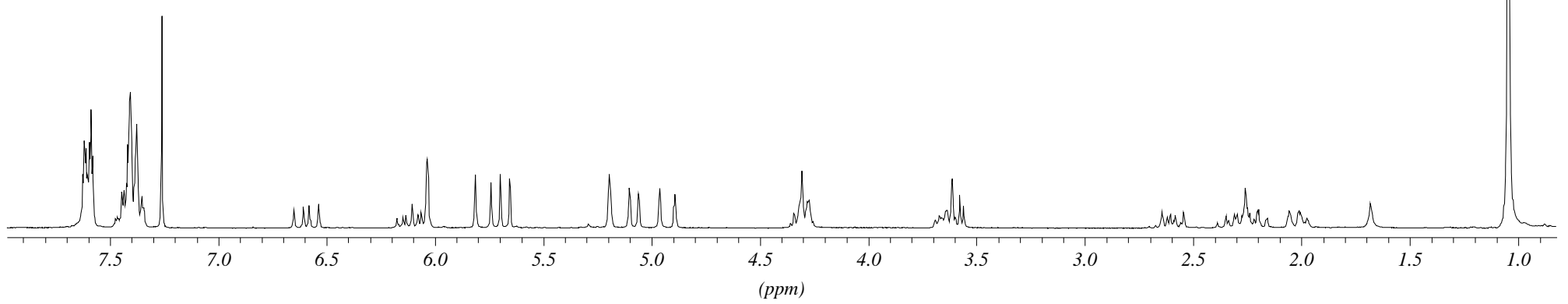




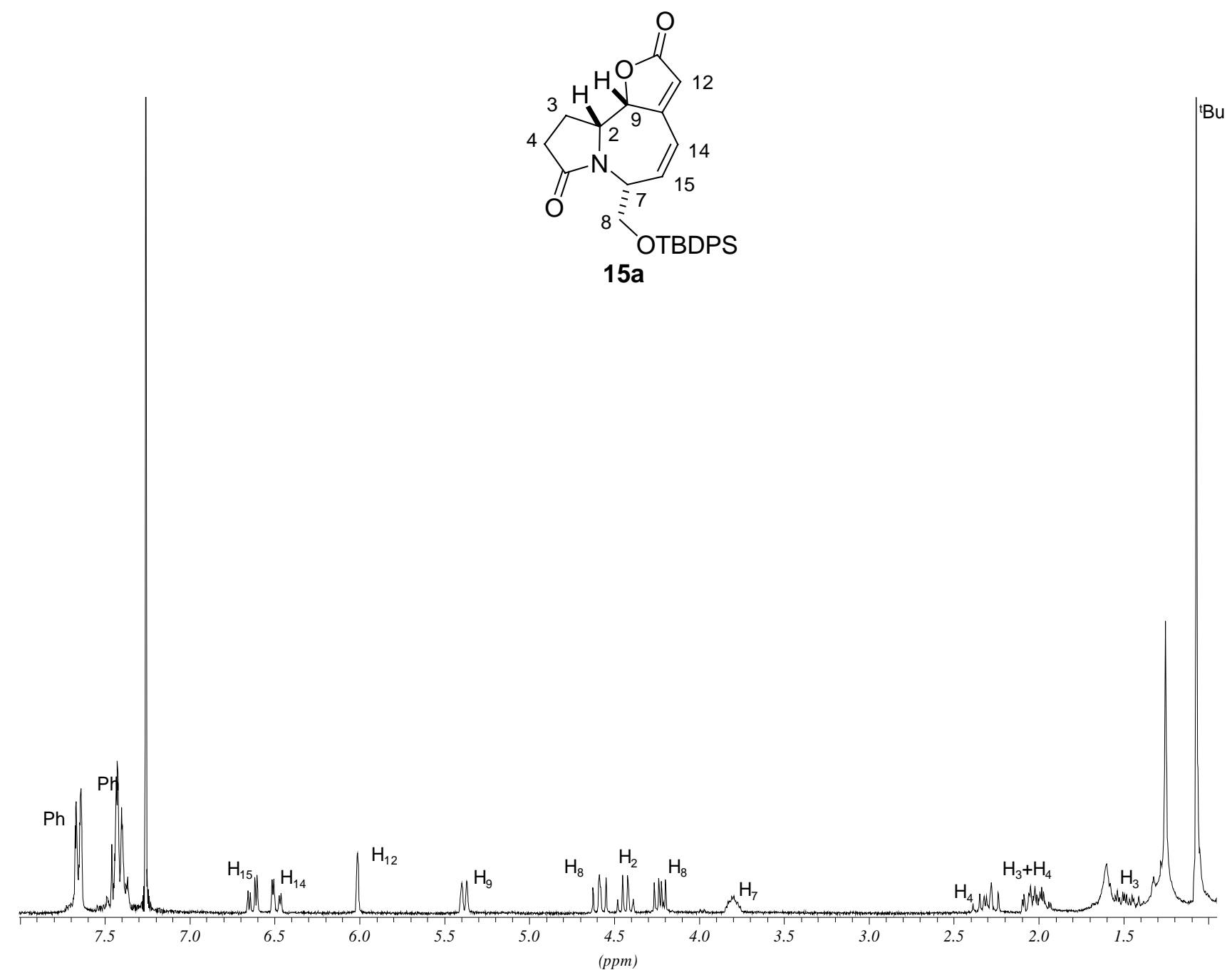




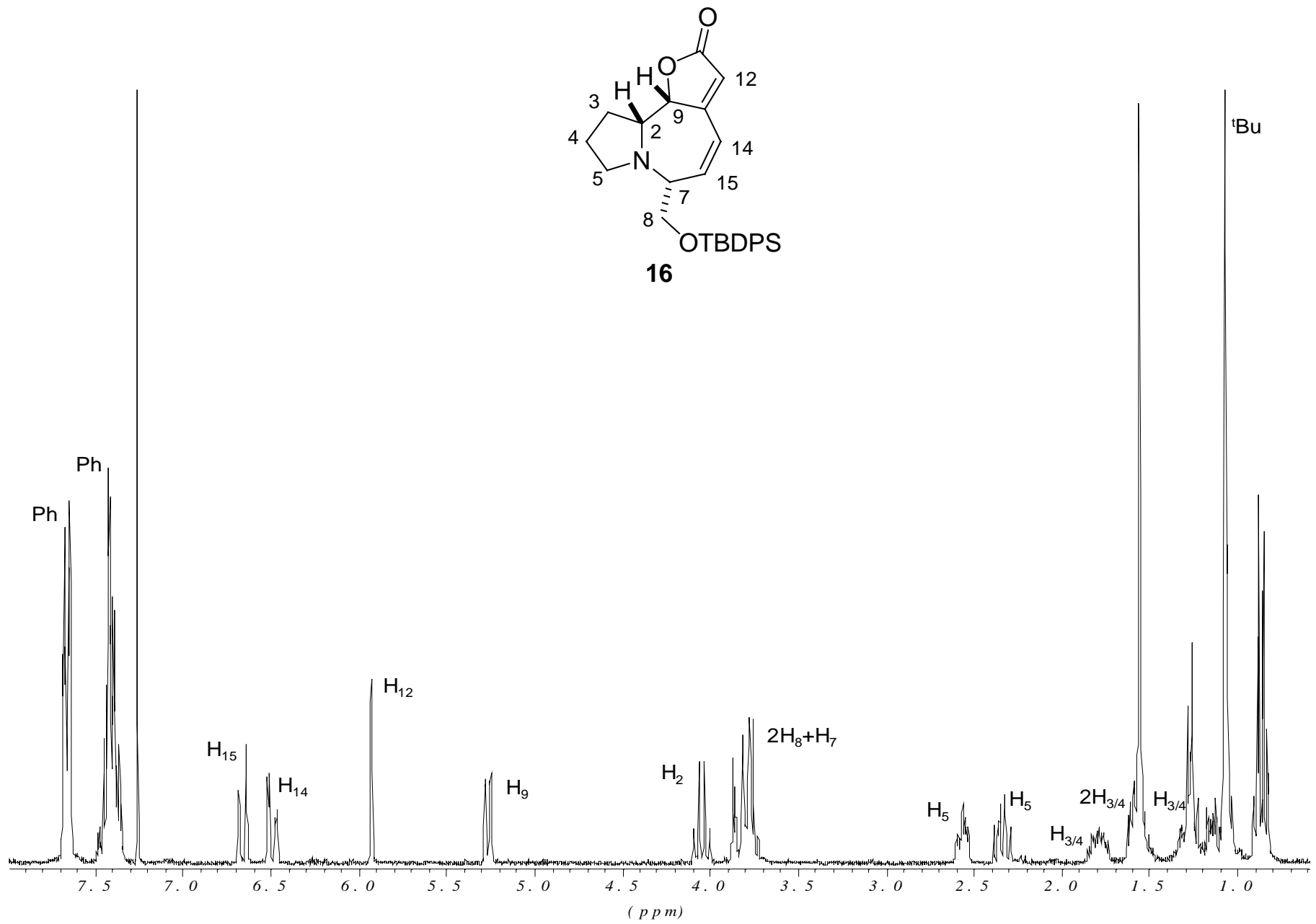




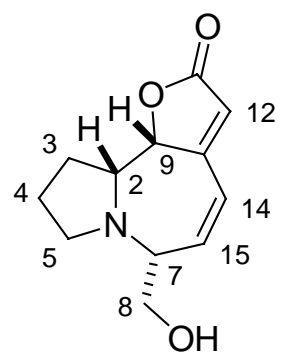

17

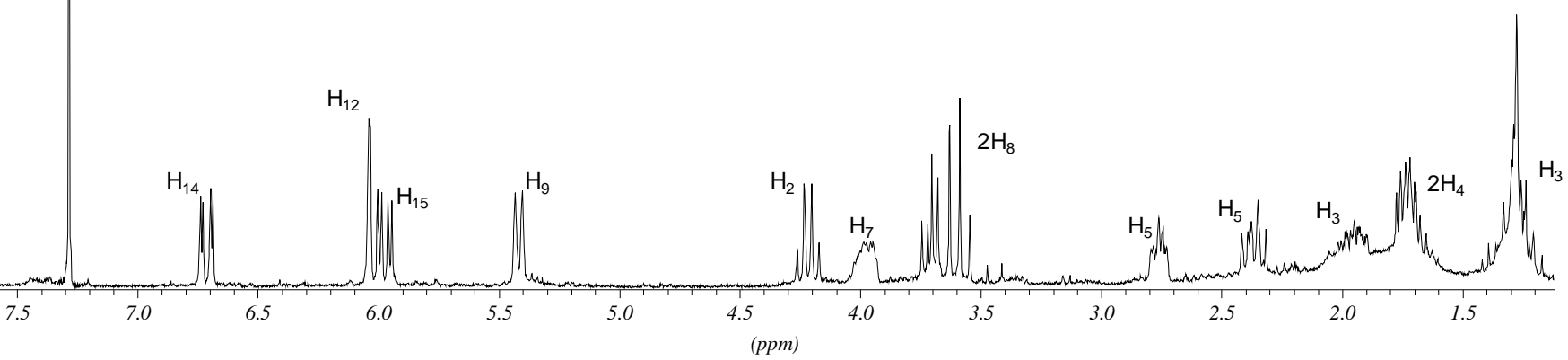




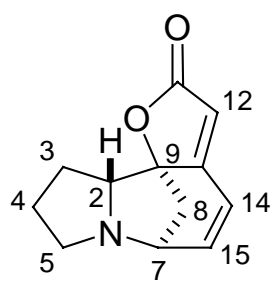

$(-)-3$

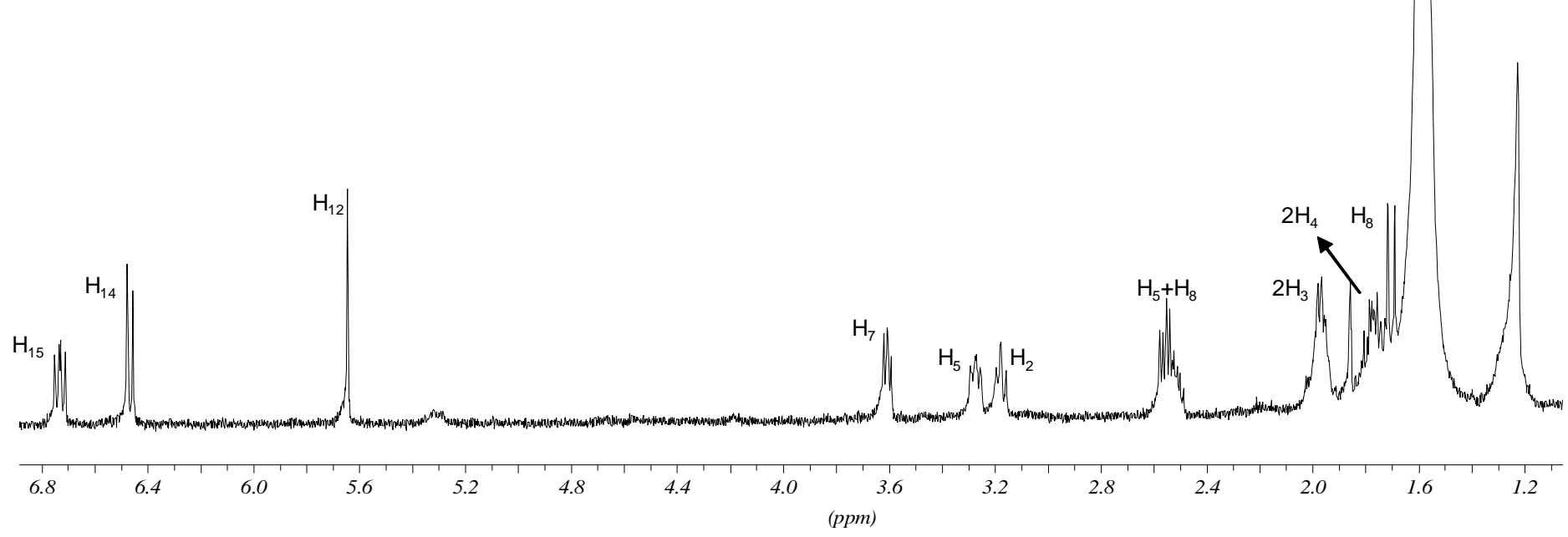



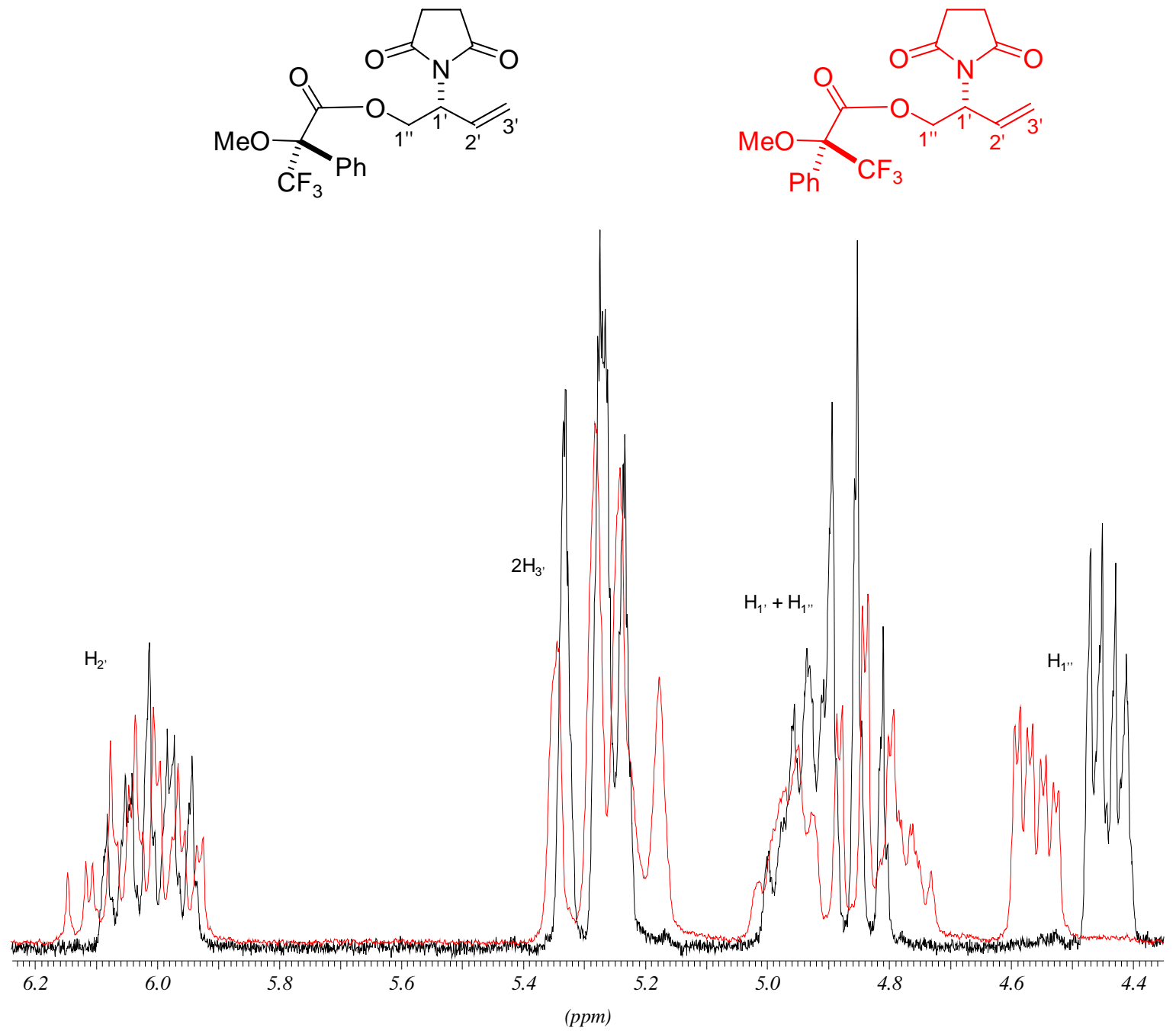